EPJ Web of Conferences 59, 15002 (2013)

DOI: $10.1051 /$ epjconf/20135915002

(C) Owned by the authors, published by EDP Sciences, 2013

\title{
Formation of counterstreaming plasmas for collisionless shock experiment
}

T. Ide ${ }^{1}$, Y. Sakawa ${ }^{2}$, Y. Kuramitsu' ${ }^{2, a}$, T. Morita $^{2}$, H. Tanji ${ }^{1}$, K. Nishio ${ }^{3}$, M. Kuwada ${ }^{3}$, H. Ide $^{3}$, K. Tsubouchi ${ }^{1}$, S. Shimazaki ${ }^{4}$, T. Taguchi ${ }^{4}$, C. Gregory ${ }^{5}$, A. Diziere ${ }^{5}$, M. Nakatsutsumi ${ }^{5}$, M. Koenig ${ }^{5}$, N. Ohnishi ${ }^{6}$ and H. Takabe ${ }^{2}$

1 Graduate School of Engineering, Osaka University, 2-1 Yamadaoka, Suita, Osaka 565-0871, Japan

2 Institute of Laser Engineering, Osaka University, 2-6 Yamadaoka, Suita, 565-0871 Osaka, Japan

${ }^{3}$ Graduate School of Science, Osaka University, 1-1 Machikaneyama-cho, Toyonaka, Osaka 560-0043, Japan

${ }^{4}$ Department of Engineering, Setsunan University, 17-8 Ikedanaka-machi, Neyagawa City, Osaka 572-8508, Japan

5 Laboratoire pour l'Utilisation des Lasers Intenses, UMR 7605, CNRS - CEA - Université

Paris VI - Ecole Polytechnique, 91128 Palaiseau Cedex, France

${ }^{6}$ Department of Aerospace Engineering, Tohoku University, 6-6-01 Aramakiazaaoba, Aoba-ku, Sendai 980-8579, Japan

\begin{abstract}
Process of counterstreaming plasma generation for laser irradiation of the innner-surface of the first plane of a double-plane target is investigated. The image taken by streaked self-emission optical pyrometer and radiation hydrodynamic simulation show the plasma from the second plane is ablated by radiation almost at the laser timing. After $\sim 5 \mathrm{~ns}$, increase in brightness and the generation of a plasma on the second plane are observed. According to the contemporary measurement of streaked interferometry, this is caused by the ablation of the second plane by the first plane plasma.
\end{abstract}

\section{INTRODUCTION}

The physics of cosmic ray acceleration is one of the most important problems in astrophysics. It is considered that cosmic rays whose energy is less than $10^{15} \mathrm{eV}$ are accelerated in collisionless shock waves in supernova remnants in our galaxy according to observation of X-ray emission. Therefore, collisionless shock waves are important in astrophysics, and they have been investigated not only theoretically and numerically but also experimentally. Previously, double-plasma devices were employed to study collisionless shock waves with relatively low Mach number $(M<2)$ and slow flow speed [1-3].

Recently, experimental researches have been carried out using high power laser systems [4-7]. A bow shock has been generated by placing a solid obstacle in the path of a high-velocity laser ablation plasma [4]. Formation of high-Mach number collisionless electrostatic shock wave in counterstreaming plasmas using a double-plane target has been reported $[6,7]$. It is essential to generate the plasmas that

\footnotetext{
ae-mail: kuramitsu-y@ile.osaka-u.ac.jp
}

This is an Open Access article distributed under the terms of the Creative Commons Attribution License 2.0, which permits unrestricted use, distribution, and reproduction in any medium, provided the original work is properly cited. 
(a)

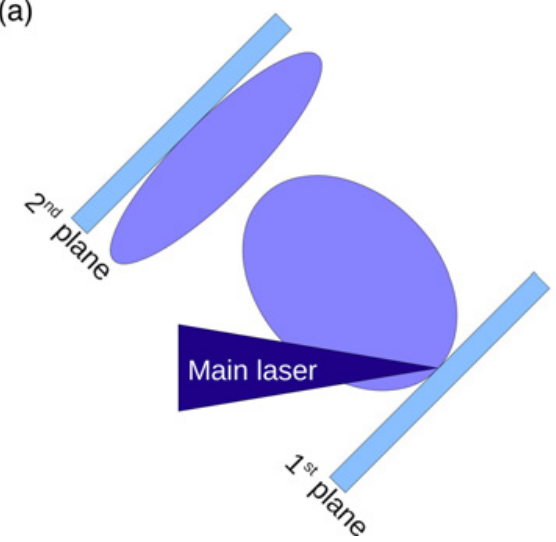

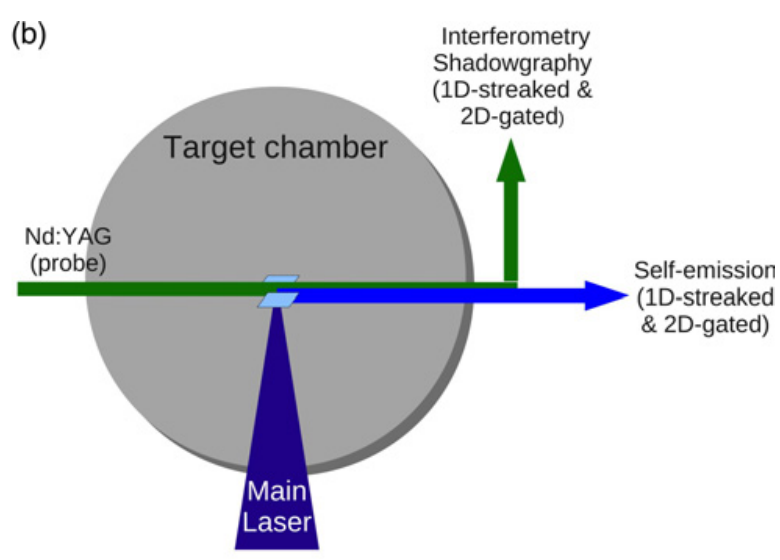

Figure 1. (a) Schematic view of the target design. A main laser beam is focused on the first plane. Both planes are made of $\mathrm{CH}$ (plastic). (b) Experimental setup. The green lines are the paths of probe YAG laser and Thomson scattering. The blue line from target chamber center (TCC) is the path of self-emission at the wavelength of $450 \mathrm{~nm}$.

stream in opposite direction in collisionless shock experiment. However, the generation mechanism of counterstreaming plasmas is not discussed precisely in these previous studies.

In this paper, we show that counterstreaming plasmas are generated irradiating double-plane target with a laser pulse, and discuss the process of generation of counterstreaming plasmas.

\section{EXPERIMENT}

The experiment was performed using Gekko XII HIPER laser system at Institute of Laser Engineering, Osaka University. The target configuration is shown in Fig. 1(a). We put two planes made of $\mathrm{CH}$ in parallel with a separation of $4.5 \mathrm{~mm}$. The dimensions of the planes were $3 \mathrm{~mm} \times 3 \mathrm{~mm}$ and $\sim 200 \mu \mathrm{m}$ in thickness. The laser delivered a 500-ps-duration (FWHM) pulse with energy of $\sim 120 \mathrm{~J}$ at a wavelength of $351 \mu \mathrm{m}$. One of nine beams was focused on an inner surface of the first plane with $300 \mu \mathrm{m}$ in diameter $\left(3.4 \times 10^{14} \mathrm{~W} \mathrm{~cm}^{-2}\right)$. Figure 1 (b) shows a schematic view of the experiment setup. $\mathrm{A} \sim 10-$ ns duration $\mathrm{Nd}$ :YAG laser diagnosed the plasma between the two $\mathrm{CH}$ planes. The probe laser was frequency converted to a wavelength of $532 \mathrm{~nm}$. Using the probe laser we obtained two-dimensional information of density by shadowgraphy with intensified charge coupled device (ICCD) cameras (gate width: $200 \mathrm{ps}$ ), and Nomarski interferometry with a gated optical imager (GOI) (gate width: $250 \mathrm{ps)}$. We also measured one-dimensional time evolution of plasma by streaked interferometry using a streak camera. We obtained two-dimensional image of self-emission using an ICCD camera (gate width: 1.6ns), and one-dimensional time-evolution using streaked optical pyrometry (SOP). The self-emission is filtered with a $450 \mathrm{~nm}$ interference filter.

\section{RESULTS}

Figure 2(a) shows a streaked interferogram. The position $x=0 \mathrm{~mm}$ corresponds to the position of the inner surface of the first plane, and that of the second plane is at $x=4.5 \mathrm{~mm}$. Initially, the fringes are static until $t=0 \mathrm{~ns}$ because there is no plasma expansion. At $t=0 \mathrm{~ns}$, they begin to move in order of distance from the first plane. This means a plasma is expanding from the first plane as a result of irradiation by a laser beam. From the timing of the fringe shift and distance $x$ of each fringe, flow velocity of the ablation plasma from the first plane is calculated to $\sim 1000 \mathrm{~km} \mathrm{~s}^{-1}$. Using the relation, 

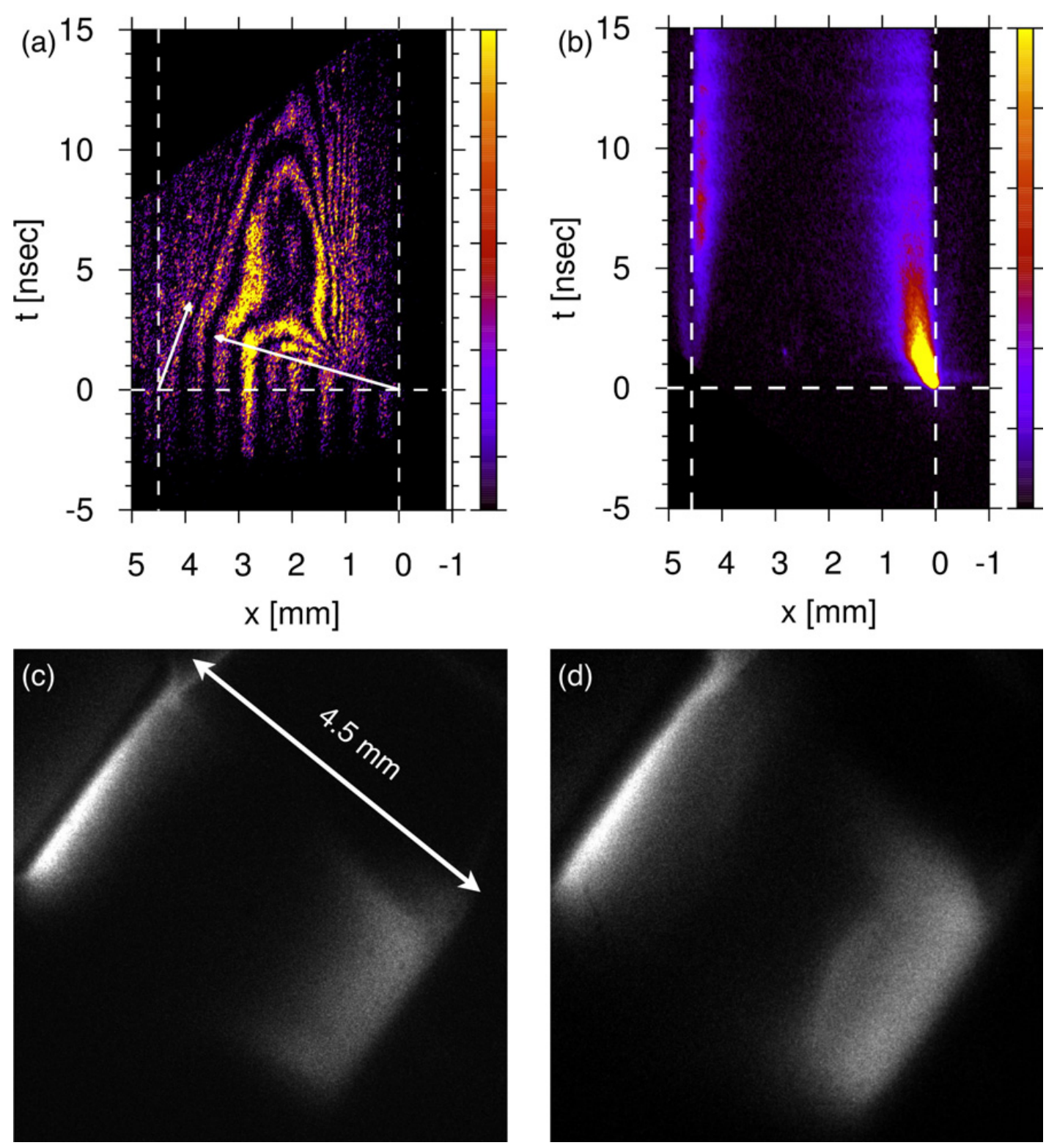

Figure 2. (a) Streaked interferogram, where $t=0$ corresponds to laser timing and $x=0$ is the position of the surface of the first plane. (b) SOP image taken on the same shot as (a). Counts correspond to the intensity of the self emission gated at the wavelength of $450 \mathrm{~nm}$. (c) and (d) Two dimensional self-emission images taken on the same shot as the streak images at $10 \mathrm{~ns}$ and at $20 \mathrm{~ns}$ from main laser irradiation, respectively.

$\frac{\Delta \phi}{2 \pi}=(L-n L) / \lambda$, where $\Delta \phi$ is phase shift, $L$ is the length of plasma along the line of sight, $n$ is the refractive index, $\lambda$ is the probe laser wavelength, $n$ is expressed using the electron density $n_{\mathrm{e}}$ and the critical density $n_{\mathrm{c}}$ corresponding to $\lambda$ as $n=\sqrt{1-\frac{n_{\mathrm{e}}}{n_{\mathrm{c}}}}$. If $n_{\mathrm{e}} \ll n_{\mathrm{c}}$, then $\frac{\Delta \phi}{2 \pi}=L\left[1-\left(1-\frac{1}{2} \frac{n_{\mathrm{e}}}{n_{\mathrm{c}}}\right)\right]$, i.e., $\frac{\Delta \phi}{2 \pi}=\frac{L}{2 \lambda} \frac{n_{\mathrm{e}}}{n_{\mathrm{c}}}$. Assuming $L \sim 1 \mathrm{~mm}$, the density of the fast ablation plasma is $\sim 2 \times 10^{18} \mathrm{~cm}^{-3}$.

Figure 2(b) shows an image of SOP taken on the same shot as Fig. 2(a). The surfaces of the first and second planes are at $x=0$ and $x=4.5 \mathrm{~mm}$, respectively. The laser pulse comes at $t=0 \mathrm{~ns}$. At the laser 
EPJ Web of Conferences

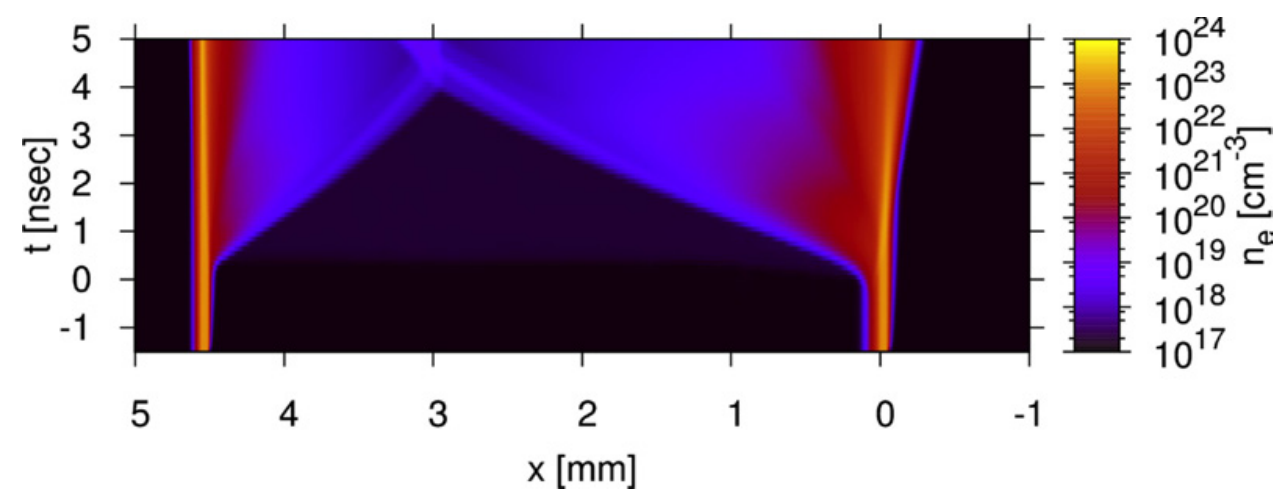

Figure 3. The time evolution of electron density distribution along the axis between the centers of the target planes.

timing, very strong emission of plasma is observed near the first plane as a result of laser irradiation and it remains bright for $\sim 5 \mathrm{~ns}$. In contrast to streaked interferometry, SOP measurement does not detect a fast plasma flow of $\sim 1000 \mathrm{~km} \mathrm{~s}^{-1}$ from the first plane because the density of the plasma is low and the streak camera can not detect self-emission of the fast plasma.

In Fig. 2(b) self-emission of the plasma near the second plane is also seen. While the laser beam irradiates only the first plane, at the laser timing there is weak emission of plasma on the second plane which is not irradiated by the laser beam. For example at $t \sim 3 \mathrm{~ns}$, the detected number of counts of self-emission from the first plane plasma is larger than that of the second plane. At $t \sim 5 \mathrm{~ns}$, the emission from second plane plasma becomes stronger. Assuming that the plasma from the first plane heats the second plane, its velocity should be $\sim 900 \mathrm{~km} \mathrm{~s}^{-1}$. It is comparable with the velocity estimated from the image of streaked interferometry.

Figures 2(c) and 2(d) show the self-emission images taken by time resolved CCD cameras on the same shot as Figs. 2(a) and 2(b). The main laser was irradiated from the left on to the right plane. At the earlier time at $10 \mathrm{~ns}$ in Fig. 2(c) we can recognize bright emissions, i.e., the plasma formations in front of both planes. At the later time at $20 \mathrm{~ns}$ in Fig. 2(d) these plasmas counter-propagate each other, and thus the counterstreaming plasma formation is confirmed from the time evolution of the two dimensional images of the self-emission of the plasmas. In Fig. 2(d) one can see a smooth shock structure in front of the right plane as seen in the previous work [7].

A two-dimensional radiation hydrodynamic (RHD) simulation was performed using PheNiX [8] code to investigate the generation and propagation of the plasmas. An Eulerian grid with cylindrical symmetry was used. The initial condition of the target is in the same way as the experimental set up. However, since the Eulerian code cannot treat vacuum, we filled tenuous CH "gas" (the mass density is $10^{-5} \mathrm{~g} \mathrm{~cm}^{-3}$ ) between the planes in the simulation. Figure 3 shows the time evolution of electron density distribution calculated by PheNiX. The ablation plasma whose density is $\sim 10^{18} \mathrm{~cm}^{-3}$ propagates from the first plane at the speed of $\sim 800 \mathrm{~km} \mathrm{~s}^{-1}$. A plasma is created at $t=0$ on the second plane. The laser energy on the first plane is transported at the speed of light and deposited on the second plane, i.e., the second plane plasma is ablated by radiation from the first plane plasma.

The line profile of temperature distribution at $t=3 \mathrm{~ns}$ is shown in Fig. 4(a). The laser beam is gaussian with $500 \mathrm{ps}$ FWHM, and the time $t=3 \mathrm{~ns}$ is $2.5 \mathrm{~ns}$ after the laser peak, hence the laser intensity is negligible at that time. As shown in Fig. 4(a), calculated temperatures of the first and second plane plasmas are $\sim 20 \mathrm{eV}$ and $\sim 7 \mathrm{eV}$, respectively. Figure 4(b) shows the line profile of Fig. 2(b) at $t=3 \mathrm{~ns}$. The brightness of the first plane plasma is about five times as large as that of the second plane plasma. This means that the plasma temperature of the first plane is higher than that of the second plane. 

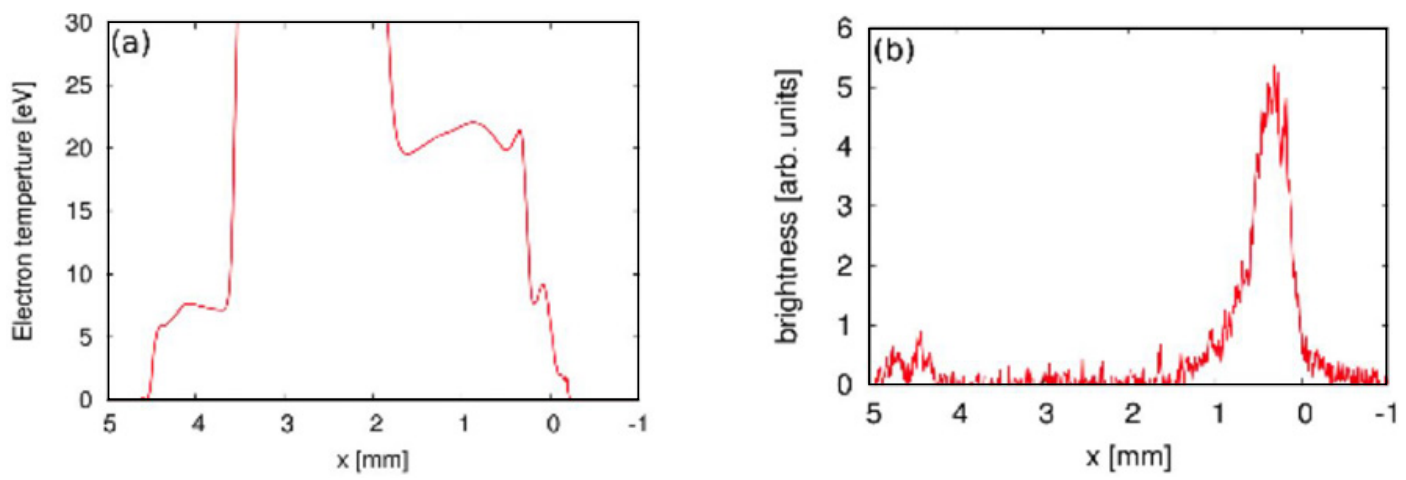

Figure 4. (a) Temperature distribution along the axis between the centers of the target planes at $t=3 \mathrm{~ns}$ in Fig. 3 . (b) The line profile of brightness in Fig. 2(b) at $t=3 \mathrm{~ns}$.

\section{CONCLUSIONS}

Counterstreaming plasmas were generated using double-plane target irradiating only the first plane. At $t<5 \mathrm{~ns}$, the plasma on the second plane was ablated by the radiation from the first plane plasma. According to SOP measurement and RHD simulation, we found the difference in temperature between the two plasmas. At $t \sim 5 \mathrm{~ns}$, generation of the second plane plasma caused by the first plane plasma was observed. In addition, the flow velocity of the first plasma estimated from streaked interferometry $\left(\sim 1000 \mathrm{~km} \mathrm{~s}^{-1}\right)$ and the velocity derived from the time delay $(\sim 5 \mathrm{~ns})$ of the increase in the second plane brightness $\left(\sim 900 \mathrm{~km} \mathrm{~s}^{-1}\right)$ are comparable. These velocities are in good agreement with the RHD simulation.

\section{References}

[1] R. J. Taylor and D. R. Baker, Physical Review Letters 24, 206 (1970)

[2] H. Ikezi, T. Kamimura, M. Kako, and K. E. Lonngren, Physics of Fluid 16, 2167 (1973)

[3] H. Ikezi, K. Mima, K. Nishikawa, and M. Inutake, Physical Review Letters 36, 794 (1976)

[4] A. R. Bell, P. Choi, A. E. Dangor, O. Willi, D. A. Bassett, and C. J. Hooker, Physical Review A 38, 1363 (1988)

[5] L. Romagnani, S. Bulanov, M. Borghesi, P. Audebert, J. Gauthier, K. Löwenbrück, a. Mackinnon, P. Patel, G. Pretzler, T. Toncian, and O. Willi, Physical Review Letters 101, 25004 (2008)

[6] T. Morita, Y. Sakawa, Y. Kuramitsu, S. Dono, H. Aoki, H. Tanji, T. N. Kato, Y. T. Li, Y. Zhang, X. Liu, J. Y. Zhong, H. Takabe, and J. Zhang, Physics of Plasmas 17, 122702 (2010)

[7] Y. Kuramitsu, Y. Sakawa, T. Morita, C. D. Gregory, J. N. Waugh, S. Dono, H. Aoki, H. Tanji, M. Koenig, N. Woolsey, and H. Takabe, Physical Review Letters, 106, 175002 (2011)

[8] N. Ohnishi, M. Nishikino, and A. Sasaki, Journal de Physique IV 133, 1193 (2006) 